\title{
The Visual IMPlications OF InSPECTION Time
}

\author{
Tyler Garaas $^{1}$ \\ tgaraas@gmail.com
}

\begin{abstract}
The quest to define human intelligence has led researchers down a large range of paths. One such path has been to search for a single psychometric measure that can be used to account for a large portion of the variance in human mental ability. Inspection Time (IT) has emerged at the forefront of these efforts and is often referred to as the amount of time required to make a single observation of sensory input. IT can be shown to account for approximately $20 \%$ of the variance in human mental ability. In this study, we attempt to gain an insight into the nature of IT as a psychometric measure by contrasting individuals that are adept at performing the IT task (those with low ITs) with individuals that are not (those with high ITs) using oculomotor and taskperformance measures recorded during two visual tasks. These tasks were designed to test participants' visualattentional control and visual working memory under varying degrees of difficulty. The data show that a sensory-level theory of IT is incapable of accounting for the results found during the visual tasks, which leads us to introduce a novel theory of IT that places IT as a measure of information propagation. A discussion is presented on the implications and need for future validation of the theory.
\end{abstract}

Keywords: Inspection time, Intelligence, Eye Movements, Visual Search

\section{INTRODUCTION}

What defines human intelligence? Is it a simple measure of processing speed? Is it based on a person's ability to extrapolate knowledge? The search for the psychological bases of human intelligence has led researchers to attempt to answer this question using a large variety of methods. One such method is to find a single elementary measure that accounts for the variance of human mental ability. Early studies found limited success using simple reaction time tasks $[15,29]$. However, these measures failed to gain support due to their accounting for only a small percentage of the variance in mental ability and their theoretical intractability in explaining how they account for variance in mental ability [4, 19, 7]. One measure that has

\author{
Marc Pomplun ${ }^{1}$ \\ marc@cs.umb.edu
}

succeeded so far where reaction time tasks had failed is that of Inspection Time (IT). IT is often described as the amount of time required for participants to make a single observation of sensory input. Since participants are allowed as long as necessary to respond to a single trial of the task used to measure IT, differences in physical ability cannot confound the measure as with the reaction time tasks. In this way, IT has a theoretical "leg up" in explaining how it accounts for the variance in mental ability. Additionally, IT was created to be so fundamental as to be "relatively immune from influence by higher cognitive activities or by motivational and social factors" [32]. Aside from its theoretical attractiveness, IT has also been shown to account for a large percentage of the variance in human intelligence; the estimated correctedcorrelation between IT and IQ is -0.50 [10]. The success of IT to both account for a large part of the variance in mental ability, along with its consistent theoretical rationale for doing so, led some researchers to initially declare that individual differences in IT cause individual differences in IQ [3, 28, 32]. However, more recently, Dreary et al. $[8,9,10]$ have expounded upon the dangers of following such a simple assumption.

In its most prevalent form, the IT task begins by cueing participants' attention by displaying a simple cue figure (Fig. 1a) in the immediate vicinity of the impending stimulus. Following the presentation of the cue figure, the actual stimulus (Fig. 1b) is presented to the user. The usual stimulus is referred to as the 'pi-figure' because of its resemblance to $\Pi$. Simply put, the figure consists of two parallel, vertical lines connected at their tops to a third, horizontal line. One of the vertical lines is extended to be longer than the other by a visual angle large enough such that, given adequate presentation time, the discrimination of which line is longer can be made perfectly by participants with a large range of mental abilities. Immediately following the presentation of the stimulus, a backward-mask (Fig. 1c) is presented in the immediate area of the preceding stimulus to prevent any processing of an iconic image. It is then the participants' task to answer as to which of the two vertical lines was longer. The participant is tested using a range of stimulus onset asynchronies (SOAs, or the time intervals that the

\footnotetext{
${ }^{1}$ Visual Attention Lab

Computer Science Department

University of Massachusetts Boston
} 
pi-figure is visible) so that it is possible to identify a critical stimulus onset asynchrony (CSOA) at which the participant achieves a preset accuracy level (e.g. $75 \%$ or $95 \%$ ). This CSOA is then operationally defined as the participant's IT.

Despite the large amount of success that has been achieved using IT, unresolved issues regarding IT still remain. For instance, the mask originally used by Vickers, Nettelbeck, and Wilson [31] has been shown to exhibit a mask-breaking effect that can be used to undermine the IT measurement $[1,11,12,13,23,14]$. This mask breaking effect was often present in the form of apparent motion, which could be used by participants to artificially improve their IT. Furthermore, at present, there is some disagreement over what IT is actually measuring. As mentioned before, participants are not under any time pressure to make a decision, and their ability to answer accurately rests solely on whether they could discriminate the longer line during the allotted presentation time. For this reason, IT has often been described as a measure of participants' speed of information intake or speed of sensory processing [31]. Some researchers have disputed this and instead claim that IT is a measure of the temporal resolution of a participant $[6,34,35]$. Still others have regarded IT as a measure of general processing speed [5]. Perhaps the most pressing unresolved matter relating to IT is the causal direction between IT and IQ. Various researchers' initial claims, that differences in IT cause differences in mental ability, have since been discarded and if IT is to remain as the foremost psychometric test used to account for variance in mental ability, this issue, above all, must be resolved. These issues demonstrate that IT is far from being fully understood as a psychometric measure.

It has been known for quite some time that oculomotor measures (measures related to eye movements) can be used to capture an insight into the underlying cognitive processes of a person. One common example is the use of pupil size as an indicator of cognitive load. Aside from pupillary changes caused by environmental factors, consistent changes in pupil size have also been shown to be caused by processing factors such as processing difficulty and working memory load $[2,20,21,22]$. For instance, Kahneman and Beatty [21] presented participants with a string of three to seven digits at a rate of one per second. After a two-second pause, subjects were asked to repeat the string that was presented to them. It was found that pupillary diameter increased with the presentation of each digit, reaching a maximum in the pause prior to their repeating the string. During the report, pupillary diameter decreased with each digit spoken all the way to the baseline following the report of the final digit. Other oculomotor measures have also been shown to provide insight into a person's cognitive processes [16, 17, 24, 25, 27, 33].
It is our intent to apply the information and insight gained from these oculomotor measures recorded during two visual tasks towards determining the true nature of IT. The visual tasks were designed specifically to test participants' visual-attentional control and visual working memory. Specifically, participants performed a visual search task where they searched a display for a target object amid distracters that varied in color or orientation from the target and a comparative visual search task in which participants attempted to locate a difference between two spatially-separated sets of objects. Prior to participating in the visual tasks, participants' ITs were first recorded using a modified version of the standard IT task.

\section{INSPECTION TIME TASK}

To record participants' ITs, we devised an IT task modeled after the standard, backward-masked IT task with two slight changes. One; the mask used originally by Vickers, Nettelbeck, and Wilson [31] has been shown to exhibit a mask-breaking effect, and as such, we designed a mask similar to that used by Knibb to reduce this effect [23]; simply put, the mask attempts to "overload" the participant's visual field, thus preventing any processing of an iconic image. Two; the standard IT task requires participants to perform the same number of trials across a wide range of stimulus onset asynchronies (SOAs) regardless of their relevance to a particular participant. In an attempt to reduce the number of irrelevant trials (and therefore the overall length of the IT task), we have created an algorithm that actively pursues only the SOAs that are relevant to computing a participant's IT.

\subsection{Participants}

The IT task was performed with the assistance of 35 participants that were paid a $\$ 10$ honorarium for their participation. Of the 35 participants, 22 were male and 13 were female; 16 were undergraduate students, 17 were graduate students, and 2 were faculty at the University of Massachusetts Boston. The median age was 28 and ranged from ages 18 to 41 . All of the participants had intact vision and some used corrective lenses.

\subsection{Apparatus}

Stimuli were presented on a 21-inch Dell P1130 monitor using the resolution $1024 \times 768$ and a refresh rate of $120 \mathrm{~Hz}$. Participants sat approximately $60 \mathrm{~cm}$ from the screen, resulting in a horizontal and vertical viewing angle of $31.5^{\circ}$ and $24.6^{\circ}$ respectively. Participants' responses were recorded using a standard PC mouse. 


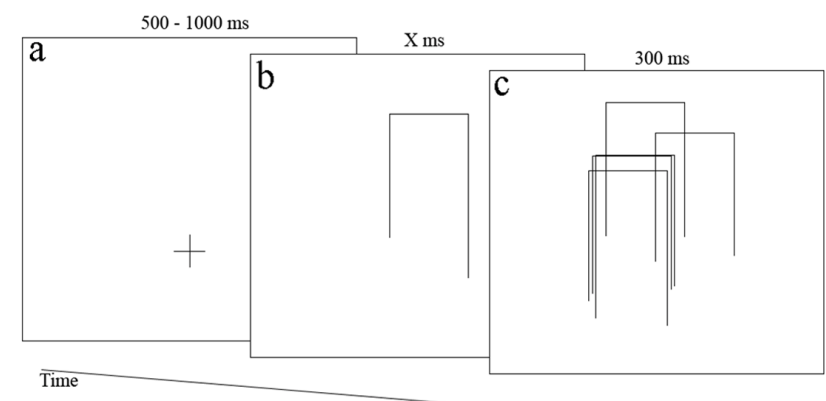

Fig. 1 IT task images: (a) the cue figure presented just prior to stimulus onset to focus attention. (b) IT stimulus, commonly referred to as the 'pi figure'. (c) the backward-mask presented after stimulus presentation to prevent any iconic-image processing.

\subsection{Materials}

The IT task stimulus, referred to as the 'Pi-figure', consisted of two vertical, parallel lines connected to a horizontal line at the top of each vertical line (Figure 1b). The Pi figure comes in two forms, one with the left, vertical line slightly longer and one with the right, vertical line slightly longer; Figure $1 \mathrm{~b}$ represents the latter. The line lengths for the target-stimulus are $3.4^{\circ}, 5.1^{\circ}$, and $6.8^{\circ}$ for the horizontal, short-vertical, and long-vertical lines respectively. To focus participants' attention, a simple cue in the form of a cross was presented immediately prior to stimulus onset (Figure 1a). Immediately following presentation of the stimulus, a backward-mask was presented to disrupt any processing of an iconic image. The mask was composed of five Pi-figures randomly placed in the immediate area of the previous stimulus (Figure 1c).

\subsection{Procedure}

Prior to the start of the experiments, each participant was given instructions about their task. To accustom participants with the task, six initial practice trials were performed starting with extremely large SOAs and leading to moderately low SOAs. During the first practice trial, the IT stimulus was presented to the participant for $750 \mathrm{~ms}$. The following 5 practice trials presented the target-stimulus for $750 \mathrm{~ms}, 750 \mathrm{~ms}, 525 \mathrm{~ms}$, $300 \mathrm{~ms}$, and $150 \mathrm{~ms}$ respectively; the first experimental trial was then presented for $75 \mathrm{~ms}$. Prior to every stimulus presentation, the cue figure was presented for a random period of time between $500 \mathrm{~ms}$ and $1000 \mathrm{~ms}$. Immediately following the presentation of the stimulus, the backward-mask was presented for $300 \mathrm{~ms}$.

Participants were instructed to focus on the accuracy of their response and to take as long as they needed to make their response. As they were instructed, the participants pressed the left or right mouse button to indicate they believed the left or right vertical line was longer respectively. Following a participant's response, the next cue-stimulus-mask triplet was presented. The stimulus presentation period varied with the accuracy of a participant's responses in such a way that SOAs, which were consistent for two cycles of the cue-stimulus-mask triplet, were increased by $8.3 \mathrm{~ms}$ if the participant responded incorrectly to one or both trials and were decreased by $8.3 \mathrm{~ms}$ if the participant responded correctly to both. This process continued until two SOA periods could be identified; one in which the participant responded correctly $\geq 75 \%$ of the trials, and one in which the participant responded correctly $\leq 75 \%$ of the trials with at least 36 trials for each. The participant's IT was then operationally defined by using linear interpolation to estimate the time at which the participant responded correctly for exactly $75 \%$ of the trials.

\subsection{Results}

Inspection times were obtained for all but one participant, whose response accuracy fluctuated too greatly due to not fully understanding the task; this participant was excluded from Experiments 2 through 4. Recorded ITs varied from $33.3 \mathrm{~ms}$ to $158.3 \mathrm{~ms}$ with a mean of $80.1 \mathrm{~ms}$ and a standard deviation of $23.4 \mathrm{~ms}$.

To analyze differences between participants with low and high ITs, two groups of 15 were formed from the 34 participants that participated in Experiments 2 through 4 (middle four were excluded from analysis to allow for a small gap between IT groups). The low-IT group had ITs that ranged from $33.3 \mathrm{~ms}$ to $73.9 \mathrm{~ms}$ with a mean of 62.0 $\mathrm{ms}$ and a standard deviation of $9.0 \mathrm{~ms}$. The high-IT group had ITs that ranged from $81.9 \mathrm{~ms}$ to $158.3 \mathrm{~ms}$ with a mean of $98.0 \mathrm{~ms}$ and a standard deviation of $21.4 \mathrm{~ms}$.

\section{VISUAL SEARCH TASK}

Visual searches are a large part of everyday life for most of us; in fact, we perform visual searches so often that most of the time we do not even realize that we are doing so. Visual search tasks have consequently become a prominent paradigm used to gain insight into our visual attention system [30,36]; see Wolfe [36] for a review of visual search. In the visual search task presented, participants searched a display for a black, horizontal bar among a set of white, vertical and white, horizontal distracters (color feature-search), or white, vertical and black, vertical distracters (orientation feature-search), or white, horizontal and black, vertical distracters (conjunctive-search). Loosely speaking, in the featuresearch conditions, the target object seems to "pop out" from the distracter objects. The target object in the conjunctive-search condition, on the other hand, is not as 
readily locatable as the one in the feature-search conditions. We consequently predict that participants will, on average, be significantly better at locating the target object in the feature-search conditions after a single fixation than in the conjunctive-search condition. We will verify this by measuring the distance from the gazeposition to the target after a single saccade has been made. Furthermore, it could be that having a low-IT allows participants to process more items within a single fixation, which should also show through this measure.

\subsection{Participants \& Apparatus}

The 34 participants that finished the IT task participated in the visual search task. Stimuli were presented on the same monitor that was used for the IT task using the same resolution and refresh rate. Participants were seated the same distance from the monitor as in the IT task yielding the same viewing angles. Eye movements were recorded using the SR Research Eye-Link II eye-tracker system. The average error of visual angle in this system is $0.5^{\circ}$, and its sampling frequency is $500 \mathrm{~Hz}$. During the visual search task, responses were recorded using a handset (often referred to as a game-pad).

\subsection{Materials}

The stimulus displays used in the visual search task consisted of oriented bars measuring $2.1^{\circ}$ in length and $0.7^{\circ}$ in width, with features varying in two dimensions, color and orientation; leaving four possible object types, horizontal, black bars; horizontal, white bars; vertical, black bars; and vertical, white bars. Each stimulus display contained 40 of these objects, of which, one random object was chosen to be the target object and was swapped for a horizontal, black object. Stimulus displays were divided into three categories: color-search (Figure2a), orientation-search (Figure 2b), and conjunctive-search (Figure 2c). Objects in the colorsearch displays consisted of an equal mix of objects that differed from the target object in their color or in both their color and orientation. Orientation-search displays were identical to color-search displays except that the distracters differed from the target object in their orientation or in both their orientation and color. Conjunctive-search displays were composed of an equal mix of objects that always differed from the target object in a single dimension. Objects were randomly placed in a screen-centered display area which had a length and width of $20.7^{\circ}$; the minimum distance between object centers was $2.6^{\circ}$. All stimulus displays were generated prior to starting the experiment so that each participant was subject to the same set of displays.
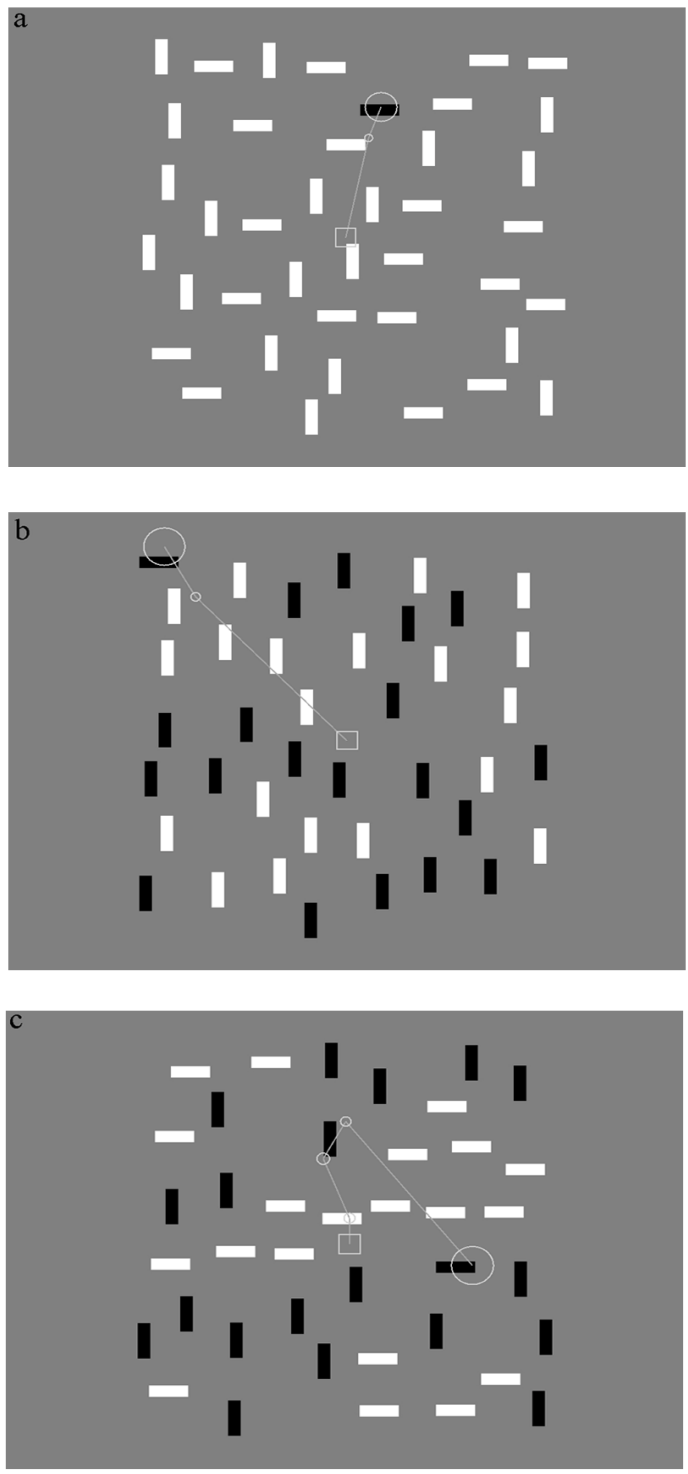

Fig. 2 Visual search task images with sample eye movements of a participant superimposed on each: (a) example colorsearch stimulus display. (b) example orientation-search stimulus display. (c) example conjunctive-search stimulus display.

\subsection{Procedure}

Participants were informed of the categories of the stimulus displays and of the identity of the target object prior to starting the experiment. They were instructed to find the target object in each trial as quickly and accurately as possible, and to then press a button on the game-pad while fixating on the target object. Prior to starting the experiment trials, participants were fitted with the eye-tracker headset, which was followed by the calibration of the eye-tracker system. Participants were 
then tested under three conditions: a color-search condition, an orientation-search condition, and a conjunctive-search condition; each condition was composed solely of displays from their respective display category. Trial conditions were presented in blocks of ten trials plus two training trials that were presented the first time a trial condition was presented. Participants were shown eight blocks of trials that were broken down into two color-search blocks, two orientation-search blocks, and four conjunctive-search blocks. The ordering of blocks and stimulus displays within each block were completely randomized, except for the two training displays, which were always presented at the start of the blocks they were present in. Prior to each trial, a simple drift correction was performed in which participants were instructed to fixate on a dot shown in the center of the screen and press a button to start the trial. Trials ended only after the button press, indicating they were fixating on the target object.

\subsection{Results}

The visual search task, as well as the comparative visual search task, generated a large amount of data, of which, only the most relevant are reported. Variables for the visual search task were analyzed using a 2-way ANOVA with IT group (low-IT vs. high-IT) as a between-subject factor and task condition (color-search vs. orientation-search vs. conjunctive-search) as a withinsubject factor. The distance between participants' fixation location at the button press and the target object, referred to as trial accuracy from here on, was $2.6^{\circ}$ with a standard deviation of $0.7^{\circ}$. To provide a baseline to compare trial accuracies against, rotated trial accuracies were also computed by rotating the display $180^{\circ}$ around its center and computing the distance between participants' non-rotated final fixation location and the target object's new location; rotated trial accuracy was $8.1^{\circ}$ with a standard deviation of $2.5^{\circ}$. The significant difference between these two accuracy measures, $\mathrm{t}(29)=$ $11.59, \mathrm{p}<0.001$, makes it clear that participants were in fact performing the task. The distance between the location of the second fixation (after the first saccade) and the target object (see Figure 3a) was significantly lower for low-IT participants $\left(5.7^{\circ}\right)$ than for high-IT participants $\left(6.5^{\circ}\right), \mathrm{F}(1 ; 28)=4.24, \mathrm{p}<0.05$. As predicted, the accuracy after the first saccade also varied significantly between task conditions (color-search: $3.1^{\circ}$; orientation pop-out: $6.2^{\circ}$; conjunctive-search: $\left.8.9^{\circ}\right), \quad \mathrm{F}(2 ; 56)=$ 148.27, $\mathrm{p}<0.001$. Relative pupil variance (see Figure $3 \mathrm{~b}$ ), which was calculated each trial as the difference between a participant's minimum and maximum pupil size divided by their minimum pupil size, varied significantly across task condition (color-search: 0.04; orientation-search: $0.06 ; \quad$ conjunctive-search: 0.11 ), a

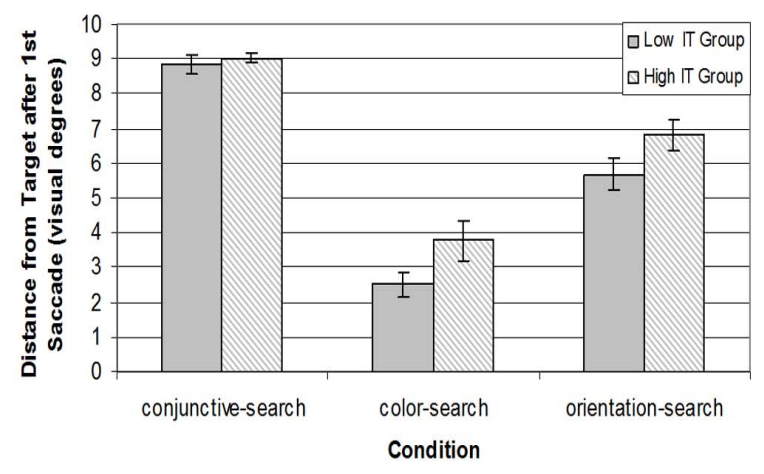

b

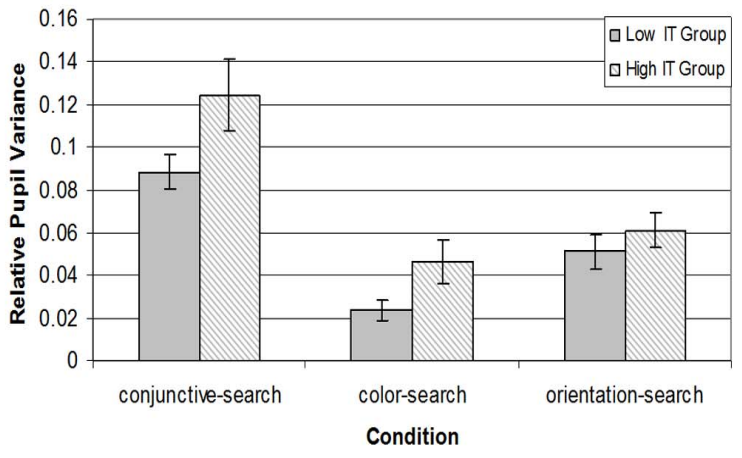

C

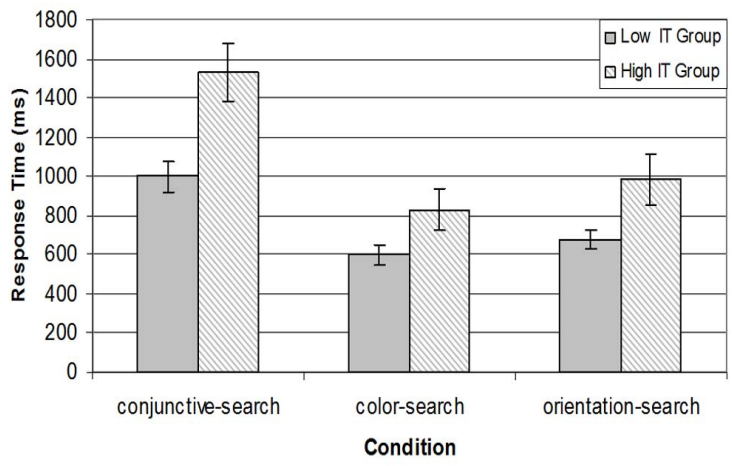

Fig. 3 Visual search task results: (a) distance to the target after the first saccade. (b) relative pupil variance. (c) response time for the low- and high-IT groups.

$\mathrm{F}(2 ; 56)=92.16, \mathrm{p}<0.001$, and tended to be smaller for participants in the low-IT group (0.05) than in the high-IT group $(0.08), \mathrm{F}(1 ; 28)=3.23, \mathrm{p}<0.1$, which implies that low-IT participants required a smaller cognitive load to complete the visual search task trials than high-IT participants.

As predicted, response times (see Figure 3c) were found to be significantly shorter for low-IT participants $(755.3 \mathrm{~ms})$ than for high-IT participants $(1114.1 \mathrm{~ms})$, 
$\mathrm{F}(1 ; 28)=7.90, \mathrm{p}<0.01$. Response times also differed significantly across task condition (color-search: 709.6 $\mathrm{ms}$; orientation-search: $828.5 \mathrm{~ms}$; conjunctive-search: $1266.0 \mathrm{~ms}), \mathrm{F}(2 ; 56)=65.15, \mathrm{p}<0.001$. Not surprisingly, the interaction between IT group and task condition did reach significance, $\mathrm{F}(2 ; 56)=9.04, \mathrm{p}<0.001$, since the separation between response times for the conjunctivesearch condition is clearly larger than it is for either of the feature-search conditions. The number of fixations per trial was also significantly lower for low-IT participants (2.3 fixations) than for high-IT participants (3.3 fixations), $\mathrm{F}(1 ; 28)=11.51, \mathrm{p}<0.005$, which comes a direct consequence of the statistically identical fixation durations between the two IT groups. The number of fixations also varied significantly across the task condition (color-search: 1.9 fixations; orientation-search: 2.4 fixations; conjunctive-search: 4.2 fixations), $\mathrm{F}(2 ; 56)=$ $104.00, \mathrm{p}<0.001$. A significant interaction between IT group and task condition that corresponds to the one for response time was also found for the number of fixations, $\mathrm{F}(2 ; 56)=9.04, \mathrm{p}<0.001$.

\subsection{Discussion}

In agreement with our hypothesis, response times were significantly shorter for low-IT participants than high-IT participants across all task conditions. Interestingly, low-IT participants also made significantly fewer fixations during each trial. This result does affirm that having a lower-IT does indeed afford a person some sort of greater visual ability, but what ability exactly? The data from this experiment seems to support low-IT participants having a greater ability to process the items. If the lower response times for low-IT participants stemmed solely from faster information retrieval, we would expect a consistent difference between IT groups in response time and no difference in trial accuracy after the first saccade. However, it appears that these measures instead reflect the perceived processing-difficulty associated with each condition, which suggests that lowIT participants are afforded a greater ability to process visual stimuli. Interestingly, low-IT participants evidenced a smaller cognitive load during the task through smaller pupillary variance, which suggests that not only are they able to process visual information faster, but doing so imposed a smaller processing load on them than it does on the high-IT participants.

\section{COMPARATIVE VISUAL SEARCH TASK}

As with visual search tasks, comparative visual search tasks rely on stringent visual-attentional control [26]. However, unlike visual search tasks, comparative visual search tasks require the effective use of visual working memory for task completion. In this way, comparative visual search tasks have been shown to yield valuable insight into our use of visual working memory and visualattentional control. For instance, Inamdar and Pomplun [18] demonstrated that participants would increase the use of their visual working memory to compensate for more costly eye movements up to their visual working memory capacity.

In the comparative visual search task presented here, participants were shown two nearly identical sets of objects (one object was dissimilar between the two sets) positioned on the left and right sides of the monitor. The two sets of objects were composed of the same oriented bars that were used in the visual search task. It was the participants' job to locate the single difference between the two sets of objects. Since the two sets of objects were setup so that participants cannot simultaneously attend to both sets at the same time, participants must first "load" their visual working memory with objects from one side of the display and then "retrieve" what they have loaded to make a comparison against the objects on the opposite side of the display. Given the nature of IT, it is possible, or even likely, that participants with a low IT can load their working memory faster than participants with a high IT, which would allow them to load more objects into memory during the same amount of time. Consequently, we predict that participants with a low IT will be significantly faster (i.e. exhibit shorter response times) at locating the difference between the two hemi-fields while exhibiting evidence of larger visual working memory loads than participants with a high IT.

\subsection{Participants and Apparatus}

The 34 participants that finished the IT task participated in the comparative visual search task. The apparatus from the visual search task served as the apparatus in the comparative visual search task as well.

\subsection{Materials}

Stimulus displays for the comparative visual search task were composed of the same oriented bars that were used in the visual search task. However, stimulus displays for the comparative visual search task were composed of two sets of objects that were displayed on opposite sides of the display. The two sides were separated by $5.5^{\circ}$ and a single black line down the center of the display. Each side contained 20 objects that were composed of an equal number of the four different object types (i.e. five of each type). Objects were placed on each side such that the minimum distance between the centers of any two objects was at least $2.6^{\circ}$. The two sides of the display were identical except for a single discrepancy. A discrepancy occurred when an object from either side had its color or 

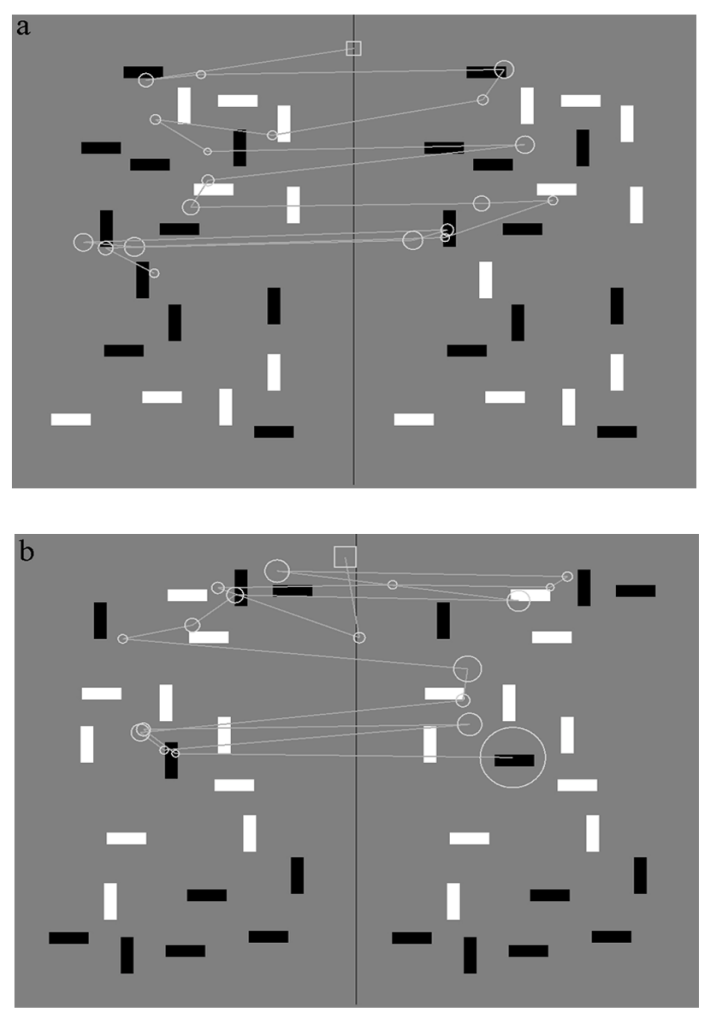

Fig. 4 Comparative visual search task images with sample eye movements of a participant superimposed on each: (a) example color-discrepant stimulus display. (b) example orientationdiscrepant stimulus display.

orientation swapped. The stimulus display categories were therefore defined by the dimension of the target object that was swapped, resulting in two categories of displays: color-discrepant displays (Figure 4a); and orientation-discrepant displays (Figure 4b). All stimulus displays were generated prior to starting the experiment so that every participant was subject to the same set of displays.

\subsection{Procedure}

Participants were given initial instructions about the nature of the experiment and their role in the task. They were instructed to locate the discrepancy between the two sides as quickly and accurately as possible, and to press a button on the game-pad while fixating on either object of the discrepancy. Participants were also instructed to serially search the display for the discrepancy starting at the top, and that if they had not located the discrepancy by the time they hit the bottom, to then search as they saw fit. Participants were tested under three trial conditions which were presented in blocks of 20 trials plus two training trials shown the first time a trial condition was presented. Prior to starting a block of trials, participants were shown a string of text that was used to indicate that the dimension of the discrepancy was either color, orientation, or unspecified for the following block; this led to the three trial conditions: the uninformed condition; the informed, color-discrepant condition; and the informed, orientation-discrepant condition. The uninformed condition was composed of an equal number of displays taken from the two stimulus display categories. The two informed conditions were composed solely of displays from their respective stimulus display categories. Participants were shown four blocks of stimulus displays, of which, two blocks were uninformed, one was informed color-discrepant, and one was informed orientation-discrepant. The ordering of blocks and stimulus displays within each block were completely randomized except for the two training trials which were presented at the start of the blocks they were present in. Prior to the start of each trial, a drift correction similar to the one used in the visual search task was performed, except that the fixation point was presented at the topcenter of the screen. Trials ended only after the button press indicating they were fixating on the target object.

\subsection{Results}

Variables for the comparative visual search task were analyzed using a 3-way ANOVA with IT group (low-IT vs. high-IT) as a between-subject factor and discrepancy information (uninformed vs. informed) along with discrepancy dimension (color vs. orientation) as withinsubject factors. Trial accuracy for all participants was $3.2^{\circ}$ with a standard deviation of $1.5^{\circ}$, and the rotated trial accuracy was $17.6^{\circ}$ with a standard deviation of $1.2^{\circ}$. The significant difference between the trial accuracies, $t(29)=$ -32.913, $\mathrm{p}<0.001$, demonstrates that participants were performing the correct task.

Comparative visual search response times (see Figure 5a) were significantly shorter for low-IT participants $(6355$ $\mathrm{ms})$ than high-IT participants $(9272 \mathrm{~ms}), \mathrm{F}(1 ; 28)=15.23$, $\mathrm{p}<0.005$. As with the visual search task, low-IT participants also made significantly fewer fixations per trial in the comparative visual search task (low-IT: 24.1 fixation; high-IT: 35.0 fixations), $\mathrm{F}(1 ; 28)=18.62, \mathrm{p}<$ 0.001 . To get a better understanding of what exactly was taking place, the number of fixations per trial was divided into those made prior to reaching the target object and those made after. Since participants were instructed to compare objects in series from top to bottom, the number of prior-fixations was calculated by counting all the fixations that occurred prior to the participant reaching the target object's vertical area, which is defined as the center of the target object \pm 50 pixel-rows; the after-fixations were then defined as all other fixations. The number of prior-fixations (see Figure 5b) made per trial was significantly lower for low-IT participants (14.0 fixations) than for high-IT participants (18.3 fixations), $\mathrm{F}(1 ; 28)=$ 
$10.86, \mathrm{p}<0.005$. As with the prior-fixations, the number of after-fixations (see Figure 5c) was also significantly lower for low-IT participants (10.2 fixations) than for high-IT participants (16.6 fixations), $\mathrm{F}(1 ; 28)=11.42$, $\mathrm{p}<$ 0.005 .

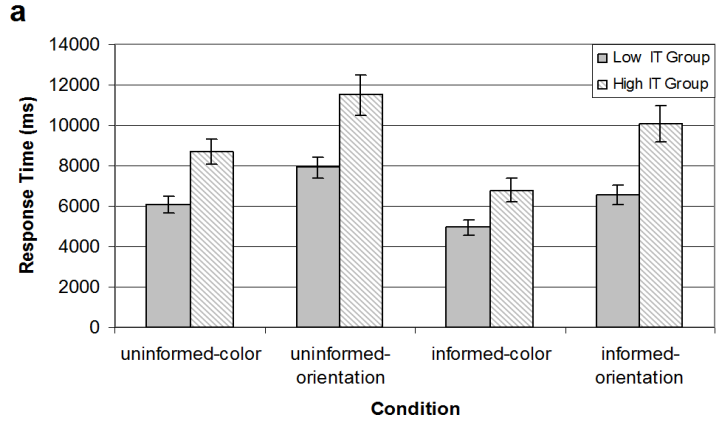

b

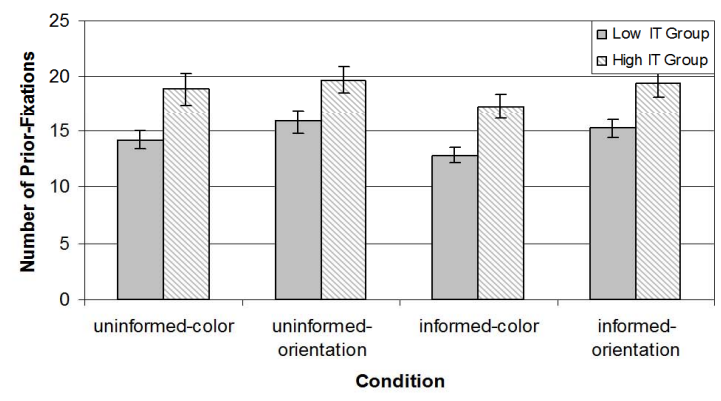

C

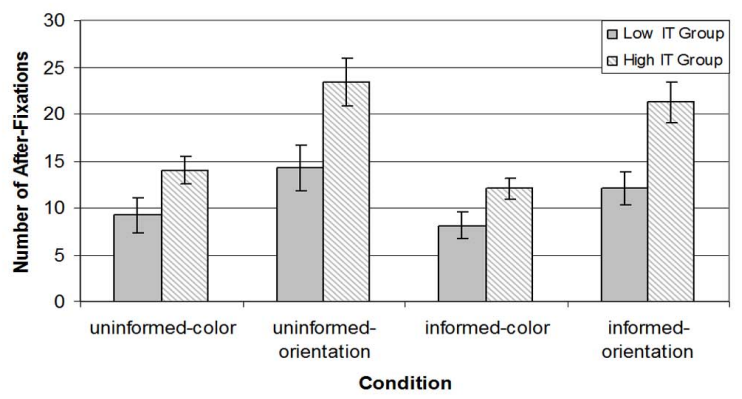

d

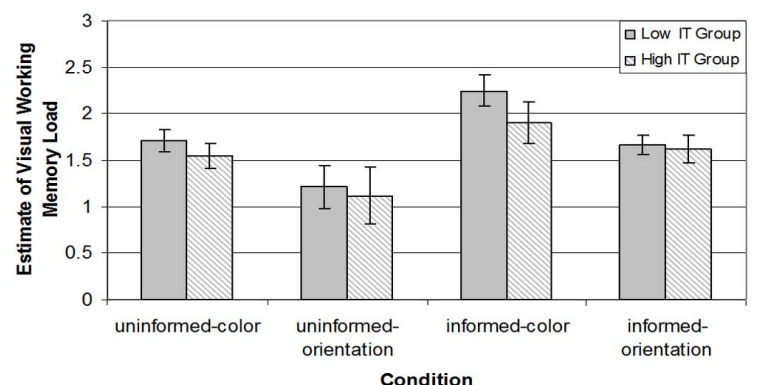

Fig. 5 Comparative visual search task results: (a) response time for the low- and high-IT groups. (b) number of priorfixations. (c) number of after-fixations. (d) estimate of visual working memory load.
The estimate of participants' visual working memory load was computed by dividing the number of objects above the target by the number of saccades that crossed the center-line prior to a participant reaching the target object's vertical area. While the visual working memory load estimate (see Figure 5d) did not vary significantly across IT group (low-IT: 1.8 objects; high-IT: 1.7 objects), $\mathrm{F}(1 ; 28)<1.0$, it did vary significantly across discrepancy dimension (color: 1.9 objects; orientation: 1.7 objects), $\mathrm{F}(1 ; 28)=7.20, \mathrm{p}<0.05$, and discrepancy information (uninformed: 1.7 objects; informed: 1.9 objects), $\mathrm{F}(1 ; 28)=6.34, \mathrm{p}<0.05$.

\subsection{Discussion}

As with the visual search task, low-IT participants were able to complete the task more quickly and with fewer fixations than high-IT participants across all conditions of the comparative visual search task. In particular, low-IT participants exhibited significantly fewer fixations both prior-to and after reaching the area of the target. That low-IT participants require fewer saccade prior to reaching the target object implies that low-IT participants can either load objects into their visual working memory more efficiently, retrieve objects from their visual working memory more efficiently, or both; exactly which, or if both, cannot be determined by this particular task since participants are free to load and retrieve objects in any arbitrarily descending order. Furthermore, that participants require fewer fixations after reaching the target object suggests that low-IT participants either recognize the target object quicker, miss the target less often, or both. Interestingly enough, the estimate of visual working memory load was not larger for low-IT participants as we had predicted, even though low-IT participants can clearly load or retrieve objects faster than high-IT participants.

\section{CONCLUSIONS}

In the visual tasks that followed the IT task, we found that participants in the low-IT group possess significantly greater performance capabilities than do the participants in the high-IT group. Superior performance for the lowIT group was found in the visual search task and comparative visual search task in the form of significantly shorter response times across all task conditions. Furthermore, low-IT participants also made significantly fewer fixations per trial of each task. These results indeed provide evidence that having a lower IT implies having some sort of greater visual abilities. Unfortunately, the data obtained during this study are insufficient to pinpoint the exact nature of low-IT participants' greater visual abilities; however, the data do provide some hints about its nature. Specifically, if low-IT participants' greater 
ability was solely at the sensory level, we would expect consistent performance and fixation count differences during the visual search task. On the contrary, performance and fixation count differences between IT groups during the visual search task appear to increase with greater perceived processing difficulty for each task condition. It would seem that this must implicate that the observed greater visual abilities stem, at least partially, from post-sensory differences. At this point, one might be encouraged to simply dismiss the sensory-level theories of IT, such as the Integration Theory advocated by White et al. [34, 35]. However, despite the similarities between the IT task and our visual search task (both rely on the efficient processing of simple visual stimuli), it would be a mistake to simply apply the visual search tasks results directly toward interpreting what IT measures. On the other hand, what we can do is use the visual search task results towards designing future experiments that can be used to test the implications formed from this study.

One current theory regarding the nature of IT is the Integration theory, which states that the presentation of the backward-mask following the IT stimulus prevents participants from correctly discriminating which leg is longer because at sufficiently short SOAs, the stimulus and mask are integrated into a single sensory observation. IT is then defined as a measure of the temporal resolution for a participant; if the resolution is fine enough, the two images will not be integrated and can be perceived separately. This theory of IT, however, is incapable of accounting for the results obtained in this study. Instead, we present a different way of interpreting what IT measures which places it more so as a post-sensory measure. It is still possible, and perhaps even likely, that IT is a measure that transcends the pre- and post-sensory division, but our data only demonstrates a clear difference in post-sensory abilities.

Based on the results of the two visual tasks, we propose that IT is actually a measure of the speed at which information propagates across neural pathways. This view of IT places its measure as both pre- and postsensory. Consider the following crude, but effective analogy. In this analogy, imagine that our brain is represented by a tree (not that this is realistic but for the sake of the analogy) and that higher branches represent higher levels of cognition; information in this analogy is represented as water and comprehension as a budding leaf. To test for comprehension, we can simply draw a line through a branch of the tree (define the task), apply water (present the stimulus), and check for a bud (analyze response). In the case of the IT task, we would draw a line through a very low branch and apply water for different periods of time to determine the amount of water needed to usually produce a bud. In this case, IT is defined as how fast the water is able to traverse the tree. If the water is removed before enough of it can reach the line, no bud will be produced.
This view of IT, which we shall refer to as the watered-tree model, does not come without its own implications, which can be tested to simultaneously examine its and the Integration Theory's validity. One test that could be performed to examine the validity of the Integration Theory would be to simply increase the line thickness of the IT stimulus and backward-mask, which, if the Integration Theory is correct, should not affect the results of the IT task. Conversely, if the watered-tree model is correct, the resulting ITs should be significantly less (from the application of a greater volume of water). Another task that could be performed to test the implications of the watered-tree model would be to draw lines further along branches or on different branches; that is to say, we could define other tasks similar to the IT task that test participants using higher cognitive (e.g. which of three lines is the longest?) and diverse cognitive (e.g. which of three objects is brightest?) tasks respectively. If the watered-tree model is correct, we would expect distinct ITs for each task within each participant while the ITs for each task between participants correlate to each other. Future research will work towards following through with these variations of the standard IT task.

\section{Acknowledgements}

This work is in part sponsored by the U.S. Department of Education through a GAANN (Graduate Assistance in Areas of National Need) Ph.D. scholarship awarded to Tyler W. Garaas.

\section{References}

[1] Alexander, J. R. M. \& Mackenzie, B. D. (1992). Variations of the 2-line inspection time stimulus. Personality and Individual Differences, 13, 1201-1211.

[2] Beatty, J. (1982). Task-Evoked Pupillary Responses, Processing Load, and the Structure of Processing Resources. Psychological Bulletin, 91, 276-292.

[3] Brand, C. R. \& Dreary, I. J. (1982). Intelligence and 'inspection time'. In H. J. Eysenck (Ed.), A model for intelligence (pp. 133-148). Berlin and New York: SpringerVerlag.

[4] Brody, N. (1992). Intelligence (2nd Ed.). San Diego, CA: Academic Press.

[5] Burns, N. R., Nettelbeck, T., \& Cooper, J. (1999). Inspection time correlates with general speed of processing but not with fluid ability. Intelligence, 27, 37-44.

[6] Burns, N. R., Nettelbeck, T., White, M. (1998). Testing the interpretation of inspection time as a measure of speed of sensory processing. Personality and Individual Differences, 24, 25-39.

[7] Detterman, D. K. (1987). What does reaction time tell us about intelligence? In P.A. Vernon (Ed.), Speed of information-processing and intelligence (pp. 177-200). Norwood, NJ: Ablex 
[8] Dreary, I. J., (2001). Human intelligence differences: toward a combined experimental-differential approach. TRENDS in Cognitive Sciences, 5, 164-170.

[9] Dreary, I. J. Looking Down on Human Intelligence: From Psychometrics to the Brain. Oxford Press, 2000.

[10] Dreary, I. J. \& Stough, C. (1996). Intelligence and Inspection Time Achievements, Prospects, and Problems. American Psychologist, 51, 599-608.

[11] Egan, V. (1986). Intelligence and inspection time: Do high IQ subjects use cognitive strategies? Personality and Individual Differences, 7, 695-700.

[12] Egan, V. (1994). Intelligence, inspection time and cognitive strategies. British Journal of Psychology, 85, 305-315.

[13] Egan, V. \& Dreary, I. J. (1992). Are inspection time strategies prevented by concurrent tasks? Intelligence, 16, 151-167.

[14] Evans, G., \& Nettelbeck, T. (1993). Inspection time: a flash mask to reduce apparent movement effects. Personality and Individual Differences, 15, 91-94.

[15] Hick, W. E. (1952). On the rate of gain of information. Quarterly Journal of Experimental Psychology, 79, 349360 .

[16] Hooge, I. T. C., \& Erkelens, C. J. (1998). Adjustment of fixation duration during visual search. Vision Research, 38, 1295-1302.

[17] Hooge, I. T. C., \& Erkelens, C. J. (1996). Control of fixation duration during a simple search task. Perception \& Psychophysics, 58, 969-976.

[18] Inamdar, S. \& Pomplun, M. (2003). Comparative Search Reveals the Tradeoff between Eye Movements and Working Memory Use in Visual Tasks. In R. Alterman \& D. Kirsh (Eds.), Proceedings of the Twenty-Fifth Annual Meeting of the Cognitive Science Society, 2003 (pp. 599604). Boston, Massachusetts.

[19] Jensen, A. R. (1987). Process differences and individual differences in some cognitive tasks. Intelligence, 11, 107136.

[20] Kahneman, D. Attention and effort. Englewood Cliffs, N.J.: Prentice-Hall, 1973

[21] Kahneman, D., \& Beatty, J. (1966). Pupil diameter and load on memory. Science, 154, 1583-1585.

[22] Kahneman, D., Beatty, J., \& Pollack, I. (1967). Perceptual deficit during a mental task. Science, 157, 218-219.
[23] Knibb, K. (1992). A dynamic mask for inspection time. Personality and Individual Differences, 13, 237-248.

[24] Pashler, H. (1988). Familiarity and visual change detection. Perception \& Psychophysics, 44, 369-378.

[25] Pollatsek, A., Rayner, K., \& Bolota, D.A. (1986). Inferences about eye movement control from the perceptual span in reading. Perception \& Psychophysics, 40, 123-130.

[26] Pomplun, M., Sichelschmidt, L., Wagner, K., Clermont, T., Rickheit, G., \& Ritter, H. (2001). Comparative visual search: A difference that makes a difference. Cognitive Science, 25, 3-36.

[27] Rayner, K. (1998). Eye Movements in Reading and Information Processing: 20 Years of Research. Psychological Bulletin, 124, 372-422.

[28] Raz, N., Willerman, L., \& Yama, M. (1987). On sense and senses: Intelligence and auditory information processing. Personality and Individual Differences, 8, 201-210.

[29] Sternberg, S. (1966). High speed scanning in human memory. Science, 153, 652-654.

[30] Treisman, A., \& Gelade, G. (1980). A feature-integration theory of attention. Cognitive Psychology, 12, 97-136.

[31] Vickers, D., Nettelbeck, T. \& Wilson, R. J. (1972). Perceptual indices of performance: The measurement of 'inspection time' and 'noise' in the visual system. Perception, 1, 263-295.

[32] Vickers, D., \& Smith, P. (1986). The rationale for the inspection time index. Personality and Individual Differences, 7, 609-624.

[33] Vogel, E. K., Woodman, G. F., \& Luck, S. J. (2001). Storage of features, conjuctions, and objects in visual working memory. Journal of Experimental Psychology: Human Perception \& Performance, 27, 92-114.

[34] White, M. (1993). The inspection time rationale fails to demonstrate that inspection time is a measure of the speed of post-sensory processing. Personality and Individual Differences, 15, 185-198.

[35] White, M., (1996). Interpreting inspection time as a measure of the speed of sensory processing. Personal and Individual Differences, 20, 351-363.

[36] Wolfe, J. M. (1998). Visual Search. In H. Pashler (Ed.), Attention (pp. 13-71). Hove, England UK. 